\title{
Diversidad de Encyrtidae (Hymenoptera: Chalcidoidea) y otras familias de Hymenoptera obtenidas con trampas Malaise en el bosque tropical caducifolio de la región de Huatulco, Oaxaca, México
}

\author{
Diversity of Encyrtidae (Hymenoptera: Chalcidoidea) and other families of Hymenoptera \\ obtained with Malaise traps in the tropical dry forest in the region of Huatulco, Oaxaca, \\ Mexico
}

Beatriz Rodríguez-Velez*, Santiago Zaragoza-Caballero y José Manuel Rodríguez

Departamento de Zoología, Instituto de Biología, Universidad Nacional Autónoma de México. Apartado postal 70-153, 04510 México, D.F., México. *Correspondencia: brodriguez@ibiologia.unam.mx

\begin{abstract}
Resumen. Se estudió la diversidad de Encyrtidae y otras familias de Hymenoptera obtenidas en trampas Malaise en el bosque tropical caducifolio de Huatulco, Oaxaca, México, entre febrero del 2005 y enero del 2006. Las recolectas se llevaron a cabo durante 5 días de cada mes. La familia Encyrtidae estuvo representada por 2 subfamilias, 7 tribus, 9 géneros y 17 especies. La tribu con mayor número de géneros y especies fue Anagyrini, con 3 géneros y 3 especies, cada una de las tribus restantes presentaron 1 género y 1 especie. Metaphycus Mercet, 1917 y Rhytidothorx Ashmead, 1900 fueron los géneros con más especies; Ooencyrtus Ashmead, 1900 fue el género más abundante. El valor de riqueza obtenido con los métodos no paramétricos Jacknife 1 y Jacknife 2 fue de 31 y 45 , respectivamente. La mayoría de las especies estuvo representada por un solo individuo. Encyrtidae no mostró aparente estacionalidad. Adicionalmente, se recolectaron otras 46 familias del orden Hymenoptera distribuidas en 2 subórdenes y 11 superfamilias. La superfamilia con mayor número de familias fue Chalcidoidea y la más abundante fue Vespoidea. Se registró un número mayor de familias durante la temporada de lluvias, siendo la abundancia muy similar para ambas temporadas, lluvias y secas.
\end{abstract}

Palabras clave: diversidad, riqueza, abundancia, estacionalidad.

Abstract. The results of the study of diversity of Encyrtidae and other families of Hymenoptera, obtained by Malaise traps in the tropical deciduous forest of Huatulco, Oaxaca, Mexico, between February 2005 and January 2006, are presented. Collections were carried out during 5 days of every month. The family Encyrtidae was represented by 2 subfamilies, 7 tribes, 9 genera and 17 species. The tribe with the largest number of genera and species was Anagyrini, with 3 genera and 3 species; the remaining tribes were represented by 1 genus and 1 species, respectively. Metaphycus Mercet, 1917 and Rhytidothorx Ashmead, 1900 were the genera with more species; Ooencyrtus Ashmead, 1900 was the most abundant genus. The richness value obtained with the nonparametric methods Jacknife1 and Jacknife 2 was 31 and 45, respectively. Most species were represented by just 1 specimen. Encyrtidae showed no apparent seasonality. In addition, other 46 families of the order Hymenoptera were collected spread over 2 suborders and 11 superfamilies. The superfamily with the greatest number of families was Chalcidoidea, the most abundant was Vespoidea. More families were registered during the rainy season; abundance was very similar for both rainy and dry seasons.

Key words: diversity, richness, abundance, seasonality.

\section{Introducción}

En general, la diversidad de los himenópteros parasitoides es mayor en las regiones tropicales que en cualquier otra región del mundo (Wolda, 1983; Noyes, 1989). Paradójicamente, son también las regiones menos conocidas y las que más rápidamente están siendo transformadas, con la consecuente pérdida de su biodiversidad (Wilson, 1988). En México, los bosques tropicales secos abarcan aproximadamente 5\% del territorio nacional (Rzedowski, 1991) y a diferencia de

Recibido: 02 octubre 2008; aceptado: 02 marzo 2009 las comunidades tropicales húmedas, que comparten gran parte de su riqueza con Centroamérica, éstos presentan un gran número de endemismos (Ceballos y García, 1995). Por desgracia, son las comunidades más amenazadas del mundo (Janzen, 1988), por lo que el estudio de su diversidad para generar información que contribuya a su conservación se hace cada vez más urgente.

Entre los insectos, los encírtidos constituyen un grupo de microhimenópteros sumamente diversos (Noyes, 1989), que juegan un papel importante en las comunidades, dado que son endoparásitoides o hiperparásitoides de otros artrópodos. En ocasiones, estas avispas también pueden comportarse como depredadores, devorando las larvas o 
huevos del huésped o perforando el integumento de los mismos (Noyes y Hayat, 1994; Trjapitzin y Ruiz-Cancino, 1995). Entre sus principales huéspedes se encuentran los homópteros, aunque también utilizan ácaros, arañas, neurópteros, coleópteros, lepidópteros, ortópteros y dípteros (De Santis, 1963). Su biología les confiere una gran importancia en las comunidades naturales, dado que ayudan a regular las poblaciones de muchas especies de insectos huéspedes. A pesar de su importancia biológica y económica, este grupo ha sido pobremente estudiado, existiendo poca información para la diversidad de una localidad o comunidad natural específica en México.

Nuestro objetivo es estudiar la diversidad de Encyrtidae y otras familias de Hymenoptera en una localidad con bosque tropical caducifolio (BTC), para analizar su riqueza, abundancia y fenología.

\section{Materiales y métodos}

El material de trabajo provino de los muestreos realizados durante el desarrollo del proyecto "Diversidad de ocho grupos de Insecta; Odonata, Lycidae, Phengodidae, Lampyridae, Cantharidae, Cerambycidae (Coleoptera), Syrphidae (Diptera) y Vespidae (Hymenoptera) en tres regiones con bosque tropical caducifolio en México" (CONACyT-CONABIO 2000-COI-0258).

El área de estudio elegida, el Parque Nacional Huatulco (PNH) se ubica en el Municipio de Santa María Huatulco y comprende un polígono situado entre los 15³9'12", y $15^{\circ} 47^{\prime} 10^{\prime \prime}$ N y 9606'30" y 96²15'00" O. Tiene una superficie de 11890.98 hectáreas, de las cuales 6374.98 corresponden a la porción terrestre, cubierta en un $80 \%$ por el BTC. El estudio incluyó muestreos dentro y fuera del $\mathrm{PNH}$, comprendiendo una franja entre los $15^{\circ} 44^{\prime} 58.4^{\prime \prime} \mathrm{y}$ $15^{\circ} 48^{\prime} 58.4$ " N y $\operatorname{los} 96^{\circ} 17^{\prime} 52.8^{\prime \prime}$ y $96^{\circ} 10^{\prime} 33.2^{\prime \prime} \mathrm{O}$, a una altura de 100 a 204 metros. De acuerdo a la clasificación de Köppen modificada por García (1981), el clima de la zona es cálido húmedo tipo Awo (w) (i). La precipitación anual varía entre 1000 y $1500 \mathrm{~mm}$, casi $97 \%$ de las lluvias se presentan durante el verano (junio-octubre), con canícula entre los meses de julio y agosto. La temperatura media anual es mayor a $22^{\circ} \mathrm{C}$ y la temperatura del mes más frío es de $18^{\circ} \mathrm{C}$. Las especies dominantes en el estrato arbóreo son: Enterolobium cyclocarpum (Jaccq.) Griseb (ahuacashle), Swietenia macrophylla King (caoba), Cedrela mexicana M. Roem (cedro), Bursera excelsa $(\mathrm{H}$. B. K.) Engl.(copal), Amphiterypgium adstringens Schiede ex Schlecht (cuachalalate), Lysiloma divaricada (Jacq.) Macbride (rajador), Cochlospermum vitifolium (Willd.) Sprengel (rosa amarilla), Caesalpinia eriostachys Benth (pintadillo), Hymenaea courbaril L. (guapinol), Celtis monoica Hemsl (escobillo) y Ceiba pentandra (L.) Gaertn (ceiba).

Para recolectar los encírtidos y otros himenópteros se utilizaron trampas Malaise tipo Townes (1972), que están ideadas para capturar insectos voladores, son de intercepción (Steyskal, 1981) y han demostrado ser un método cuya eficiencia y desempeño en la captura de insectos es constante (Kitching y Stork, 2001), empleándose con gran éxito en la recolección de microhimenópteros (Noyes, 1982). En el sitio estudiado, se colocaron 6 trampas Malaise distribuidas al azar dentro del bosque, permaneciendo en el mismo sitio durante todo el periodo de muestreo. Los muestreos duraron 5 días, fueron mensuales y se realizaron durante un año. Como líquido conservador se utilizó alcohol al 70\%. El periodo de recolección fue de febrero del 2005 a enero del 2006, exceptuando el mes de mayo.

El trabajo de laboratorio comprendió el procesamiento de 62 muestras, además de determinar las especies de Encyrtidae e identificar las familias de Hymenoptera contenidas en cada una de las muestras y el montaje y etiquetado de los ejemplares. Para el montaje, las avispas se procesaron mediante deshidratación, usando alcohol de diferentes graduaciones y acetato de amilo para evitar que se colapsaran. Las especies se determinaron con ayuda de la literatura (Noyes, 1980; Noyes et al., 1997) y consulta de colecciones especializadas (Texas A\&M University). Los ejemplares estudiados en este trabajo se depositaron en la Colección Nacional de Insectos (CNIN) del Instituto de Biología, UNAM.

Los valores de riqueza y abundancia corresponden al número de especies e individuos recolectados. La diversidad, equidad y dominancia se analizaron con el índice de Shannon, usando el logaritmo natural. Estos valores se obtuvieron con el programa Past (Hammer y Ryan, 2001). Considerando que la riqueza de especies obtenida de cualquier muestra generalmente subestima su valor real de riqueza de individuos en una localidad (Chazdon et al., 1998) se utilizaron estimadores no paramétricos con el propósito de determinar qué tan cerca se estuvo de recolectar la riqueza real de los encírtidos de Huatulco. Los estimadores no paramétricos fueron Jacknife 1 y Jacknife 2, que utilizan datos de presenciaausencia y se enfocan en especies poco abundantes o raras, o sea, las que se presentan solamente en 1o 2 muestras o que tienen 1 o 2 individuos en el conjunto de muestras (Colwell y Coddington, 1994; Moreno, 2001). Los estimadores se calcularon usando el programa EstimateS 8.0 (Colwell, 2006). Las especies recolectadas en cada mes se consideraron como una muestra (11 en total).

Para el análisis fenológico de los datos la temporada de lluvias se tomó entre junio y noviembre, y la de secas 
entre diciembre y mayo.

\section{Resultados}

Riqueza de Encyrtidae. Se recolectaron 17 especies de encírtidos, 4 de las cuales no pudieron determinarse en nivel específico ni genérico, ya que los ejemplares estaban incompletos o en malas condiciones; sin embargo, se pudo observar que se trataba de ejemplares que pertenecían a diferentes especies del resto de las recolectadas. El análisis de riqueza se basó en los ejemplares que estaban en buen estado con el objeto de lograr la determinación específica. Se reconocieron 13 especies, de las cuales sólo 6 fueron determinadas.Todos los géneros determinados ya se han registrado para el estado de Oaxaca (González -Hernández, 1997).

Las 13 especies (Cuadro 1) se encuentran distribuidas en 9 géneros, uno de los cuales es nuevo, 7 tribus y 2 subfamilias. La subfamilia con mayor número de especies es Encyrtinae con 10 (77\% del total de las especies); Tetracneminae está representada con 3 especies (23\%). La tribu con mayor número de géneros y especies es Anagyrini con 3 géneros y 3 especies, el resto de las tribus presentan 1 género y 1 especie, respectivamente (Cuadro 1). Los géneros con mayor número de especies son Metaphycus y Rhytidothorax con 3 especies cada uno ( $46.1 \%$ del total de las especies). Los otros 7 géneros están representados por 1 especie cada uno (53.8\% del total de las especies) (Cuadro 1).
El valor estimado de la riqueza de especies, basado en los estimadores no paramétricos Jacknife 1 (31) y Jacknife 2 (45), fue mayor que el valor observado (17). Esto significa que sólo se recolectó entre el $37 \%$ y el $55 \%$ de la riqueza real local. La curva de acumulación de especies (Fig. 1) obtenida con estos estimadores no alcanza la asíntota.

Abundancia de Encyrtidae. Durante el año de estudio se colectó un total de 22 individuos; la distribución de las especies parece ser heterogénea y poco abundante, puesto que $88 \%$ de las especies estuvieron representadas por 1 individuo. La especie más abundante fue Ooencyrtus pityocampae con 5 individuos, le siguió Encyrtus infelix con 2, el resto de las especies estuvieron representadas sólo por 1 individuo cada una (Cuadro 1).

Diversidad de Encyrtidae. El valor del índice de diversidad (Cuadro 2) varió por mes, el más alto (1.609) se registró en febrero. Abril, agosto y septiembre mostraron una diversidad de 0 , pues sólo 1 especie fue recolectada en cada uno de estos meses. El valor del índice de equitatividad (Cuadro 2) fue muy homogéneo durante todo el año, todos los meses presentaron el valor de 1, excepto marzo con 0.9449 , valor muy cercano a 1 . El índice de dominancia (Cuadro 2) también tuvo variación durante el año de recolección; abril, agosto y septiembre son los meses que presentan el valor más alto (1), ya que sólo 1 especie fue recolectada en esos meses; febrero tuvo el más bajo (0.2) y fue el mes en el que se registró el mayor número de especies activas (5).

Fenología de Encyrtidae. El número de especies activas fue igual durante el tiempo de lluvias y el tiempo de secas,

Cuadro 1. Número de individuos y mes de recolección de los encírtidos en Huatulco, Oaxaca, México

\begin{tabular}{|c|c|c|c|c|c|}
\hline Subfamilia & Tribu & Género & Especie & Núm. & Mes \\
\hline \multirow[t]{10}{*}{ Encyrtinae } & \multirow[t]{3}{*}{ Aphycini } & \multirow[t]{3}{*}{ Metaphycus } & M. helvolus (Compere, 1926) & 1 & Jul. \\
\hline & & & M. flavus (Howard, 1881) & 1 & Jul. \\
\hline & & & M. sp. 1 & 1 & Jun. \\
\hline & \multirow[t]{3}{*}{ Bothriothoracini } & \multirow[t]{3}{*}{ Rhytidothorax } & R. marlatti Ashmead, 1900 & 1 & Ago. \\
\hline & & & $R$. sp. 1 & 1 & Jun. \\
\hline & & & $R$. sp. 2 & 1 & Mar. \\
\hline & Encyrtini & Encyrtus & E. infelix (Embleton, 1902) & 2 & Mar. \\
\hline & Microteryni & Ooencyrtus & O. pityocampae (Merced, 1921) & 5 & $\begin{array}{l}\text { Feb., Abr., } \\
\text { Jul. Sep. Oct. }\end{array}$ \\
\hline & Sin ubicación & Lamennaisia Girault, 1922 & L. sp. 1 & 1 & Jun. \\
\hline & $?$ & Género nuevo & Gen. y sp. 1 & 1 & Feb. \\
\hline \multirow[t]{3}{*}{ Tetracneminae } & \multirow[t]{3}{*}{ Anagyrini } & Anagyrus & A. pseudococci (Girault, 1915) & 1 & Feb. \\
\hline & & Gyranusoidea Compere, 1947 & G. sp. 1 & 1 & Feb. \\
\hline & & Pseudleptomastix Girault, 1915 & P. sp. 1 & 1 & Jun. \\
\hline
\end{tabular}




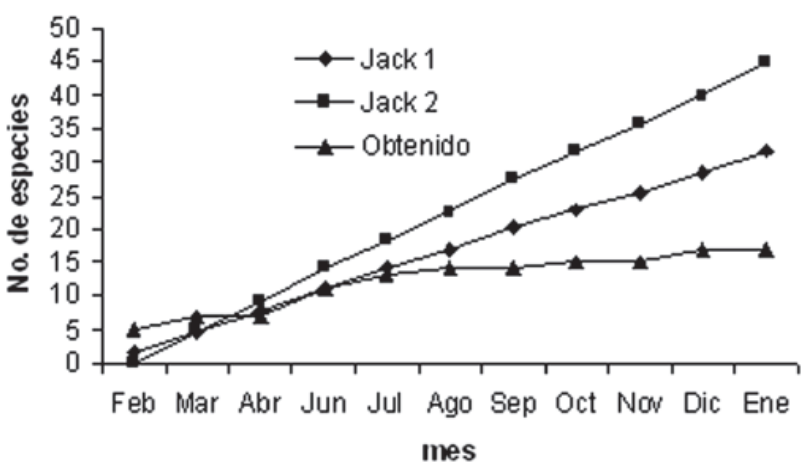

Figura 1. Curva de acumulación de especies obtenida con los estimadores no paramétricos Jacknife 1, Jacknife 2 y los valores de riqueza obtenidos.

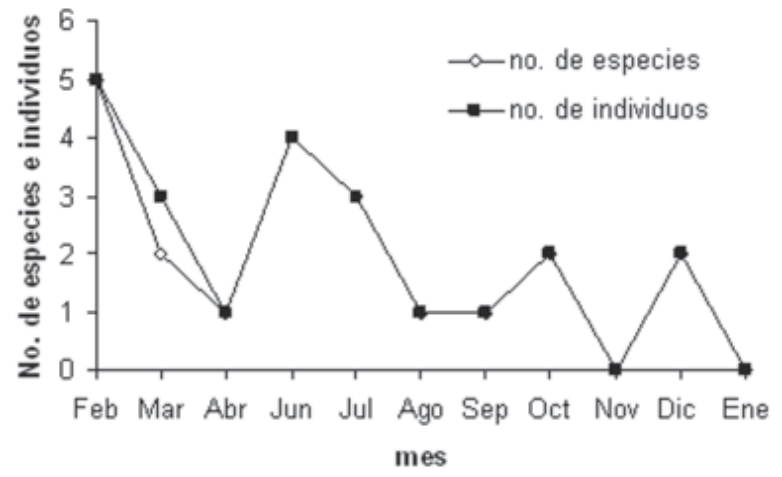

Figura 2. Número de especies e individuos de la familia Encyrtidae colectados por mes en Huatulco, Oaxaca, México.

Cuadro 2. Índices mensuales de la familia Encyrtidae de Huatulco, Oaxaca, México

\begin{tabular}{lccccccccc}
\hline & Feb. & Mar. & Abr. & Jun. & Jul. & Ago. & Sep. & Oct. & Dic. \\
\hline Diversidad & 1.609 & 0.6365 & 0 & 1.386 & 1.099 & 0 & 0 & 0.6931 & 0.6931 \\
Equidad & 1 & 0.9449 & 1 & 1 & 1 & 1 & 1 & 1 & 1 \\
Dominancia & 0.2 & 0.5556 & 1 & 0.25 & .03333 & 1 & 1 & 0.5 & 0.5 \\
\hline
\end{tabular}

9 especies para cada temporada. El mayor número de especies (5) se registró en febrero, temporada de secas, y (4) en junio, temporada de lluvias. Durante los meses de noviembre y diciembre, no se registraron encírtidos; en los meses de abril, agosto y septiembre, sólo se registró la actividad de 1 especie (Fig. 4). Ooencyrtus pityocampae (5.8\% del total de las especies) fue la única que estuvo activa durante la temporada de lluvias y la de secas. La abundancia de individuos (Fig. 2) fue igual en ambas temporadas, 11 individuos activos en cada una. El mayor número de individuos activos se registró en febrero con 5 ejemplares y en junio con 4 ; en noviembre y enero no se registró la presencia de encírtidos; en abril, agosto y septiembre se registró un individuo en cada uno de estos meses.

Riqueza de familias de Hymenoptera. Se recolectaron 46 familias del orden Hymenoptera, reunidas en 11 superfamilias y 2 subórdenes, Symphyta y Apocrita (Aculeata y Parasitica) (Apéndice 1). El suborden con el mayor número de superfamilias y familias fue Apocrita con 10 (91\% del total de superfamilias) y 44 (95.7\% del total de familias), respectivamente; de las cuales, 3 superfamilias y 19 familias pertenecen a Apocrita Aculeata, y 3 superfamilias y 25 familias a Apocrita Parasitica. El suborden Symphyta estuvo presente con 1 superfamilia (9\% del total) y 2 familias ( $4.3 \%$ del total) (Apéndice 1$)$.

La superfamilia con mayor número de familias fue
Chalcidoidea con 15 (32.6\% de la riqueza total), le siguió Vespoidea con 9(19.6\%) y Apoidea con 6(13\%), 3 familias Apiformes y 3 familias Spheciformes. Chrysidoidea estuvo presente con 4 familias; Ceraphronoidea, Evanioidea, Ichneumonoidea, Platygastroidea y Tenthredinoidea con 2 familias cada una, y Cynipoidea y Proctotrupoidea con 1 familia cada una (Fig. 3).

Abundancia de las familias de Hymenoptera. Durante el año de estudio fueron recolectados 1680 individuos con una distribución muy heterogénea de las familias, pocas fueron abundantes. La superfamilia más abundante fue Vespoidea con 1024 individuos (61\% de la abundancia total), le siguió Ichneumonoidea con 201 individuos (12\%). Chrysidoidea estuvo presente con 158 individuos (9.4\%), Chalcidoidea con 120 individuos (7.14\%), Platygastroidea con 74 individuos (4.4\%), Apoidea con 44 individuos (2.6\%), Tenthredinoidea con 19 individuos (1.1\%), Evanioidea con 16 individuos $(0.95 \%)$, Proctotrupoidea con 12 individuos $(0.71 \%)$ y Cynipoidea con 6 individuos $(0.35 \%)$ (Fig. 5). Las familias más abundantes fueron Formicidae con 653 individuos (39\%), Pompilidae con 235 (13.9\%), Ichneumonidae con 109 (6.4\%), Braconidae con $92(5.4 \%)$, Bethylidae con $87(5.1 \%)$, Dryinidae con $66(3.9 \%)$, Mutillidae con $62(3.6 \%)$, Scelionidae con 49 (2.9\%), Vespidae con 39 (2.3\%) y Pteromalidae con 34 (2\%). Estas 10 familias representan el 74\% de la abundancia total, el otro $26 \%$ está dividido en 36 familias, 


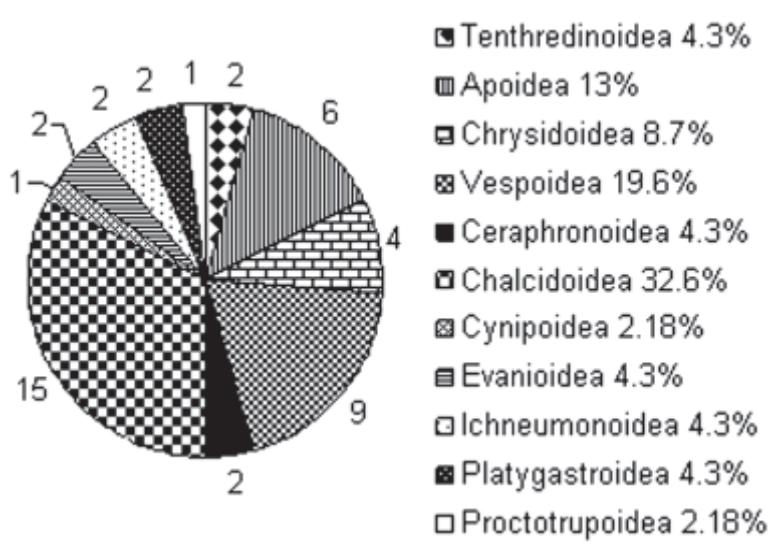

Figura 3. Número y porcentaje de las familias recolectadas en cada superfamilia en Huatulco, Oaxaca, México.

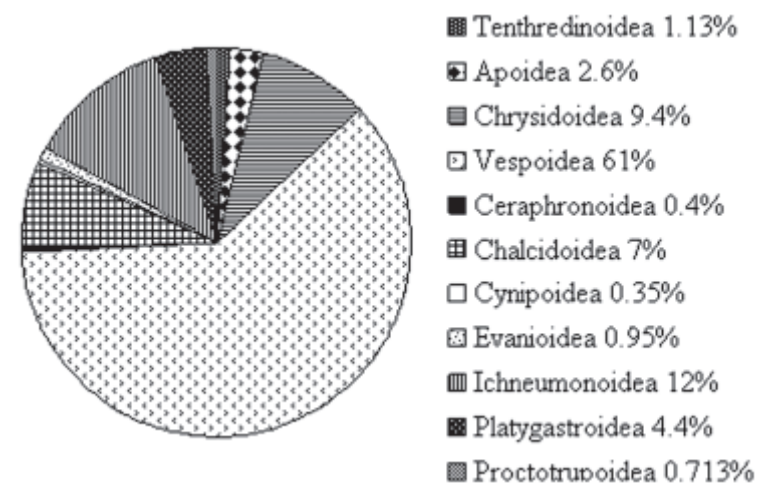

Figura 5. Porcentajes de individuos por superfamilias recolectadas en Huatulco, Oaxaca, México.

9 de las cuales están presentes sólo con 1 individuo y 5 con 2 individuos (Apéndice 1).

Fenología de las familias de Hymenoptera. La actividad de las familias varió durante el año (Fig. 4). Durante la época de lluvias se registraron 40 familias ( $87 \%$ del total), número mayor que durante la época de secas con 32 (69.5\%). En ambas temporadas se mantuvieron activas 27 familias (58.7\%) . El mayor número de familias se registró en octubre con 30 , le siguen marzo y julio con 24 familias cada uno, agosto con 22 , junio con 20 , marzo con 19 , abril y diciembre con 15 cada uno, septiembre con 14, noviembre con 12 y enero con 10 familias (Fig. 4). El mayor número de individuos que se recolectó fue en julio con 265 y el menor durante el mes de noviembre con 41 (Fig. 6). Abundancias muy similares fueron registradas para ambas temporadas, 847 individuos $(50.4 \%)$ durante la de lluvias, y $833(49.6 \%)$ durante la de secas. Diez (21.7\%) familias estuvieron activas sólo por un mes, 7 (15.2\%) por 2 meses,

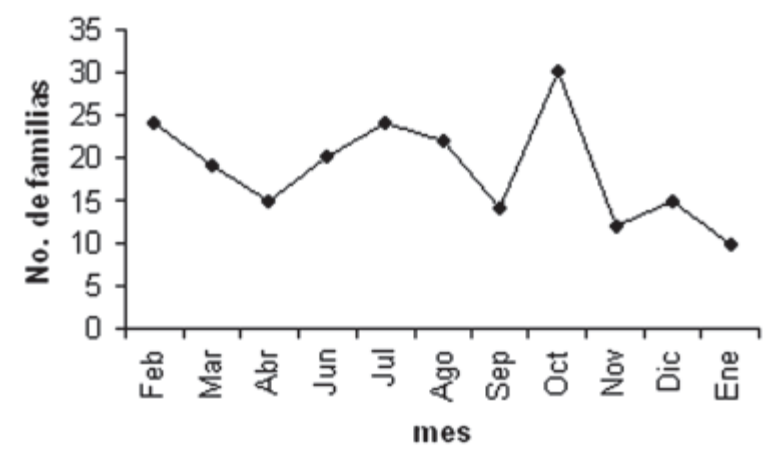

Figura 4. Número de familias activas por mes colectadas en Huatulco, Oaxaca, México.

$4(6.9 \%)$ por 3 meses, $7(15.2 \%)$ por 4 meses, $4(6.9 \%)$ por 5 meses, 2 (4.3\%)por 6 meses, $2(4.3 \%)$ por 7 meses, 2 (4.3\%) por 8 meses, 4 (6.9\%) por 9 meses, 1 (Scelionidae) (2.1\%) por 10 meses, y 3 (Formicidae, Braconidae y Pompilidae) (6.5\%) por 11 meses.

\section{Discusión}

Diversidad de Encyrtidae. Si comparamos la riqueza de especies de Huatulco, Oaxaca, con la de Huautla, Morelos, otra región con selva baja caducifolia como vegetación dominante, observamos que en ambos sitios la riqueza estimada fue mayor que la riqueza obtenida, registrándose para Huatulco entre $38 \%$ a $53 \%$ de la riqueza real para la localidad y para Huautla entre $34 \%$ a $37 \%$ de la riqueza real (Rodríguez-Velez y Woolley, 2005). El bajo número de especies recolectadas en ambas localidades muestra aparentemente una deficiencia en la forma o método de recolección, combinado a la vez con diferentes aspectos de la biología de la familia. En ambos sitios el método de recolección fue con trampas Malaise, que si bien es un buen método para la recolección de microhimenópteros, el mayor número de especies de encírtidos y calcidoideos se captura con redes de barrido (Noyes, 2008).

Es muy probable que especies raras de encírtidos con periodos cortos de vida no hayan sido recolectados, ya que su época activa como adultos no coincidió con la época de recolección (5 días por mes). La aparente rareza de algunas especies junto con sus cortas estancias (94\% de las especies de Huatulco y el $71 \%$ de Huautla estuvieron activas sólo por un mes y fueron poco abundantes, el $88 \%$ y el $65 \%$ de las especies de Huatulco y Huautla, respectivamente, estuvieron presentes con un individuo solamente), combinadas con la heterogeneidad de las selvas bajas (Trejo, 1998), disminuye la probabilidad de recolectar especies que sólo aparecen en hábitats muy 


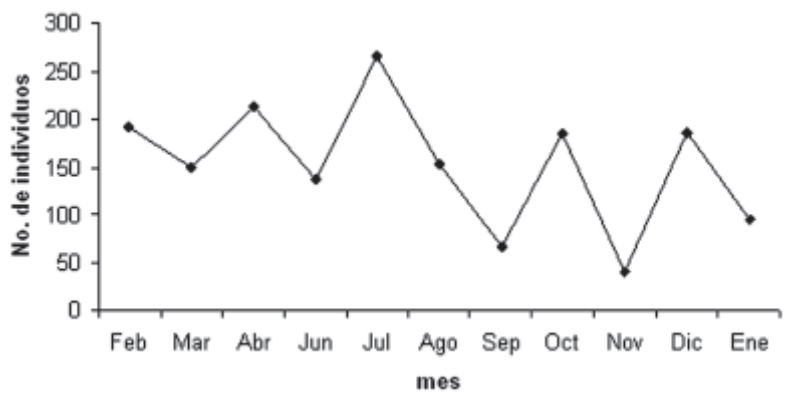

Figura 6. Número de individuos del orden Hymenoptera colectados en Huatulco, Oaxaca, México.

específicos o que están estrechamente ligadas con recursos escasos en el ecosistema, en este caso huéspedes y sus respectivas plantas de alimentación.

Tanto los encírtidos de Huatulco como los de Huautla no presentaron una aparente estacionalidad ni en riqueza ni en abundancia, lo que puede deberse a que los recursos que utilizan presentan una fenología similar, patrón que también ha sido registrado para Apoidea (Celso, 2002).

Los resultados de este estudio muestran que la familia Encyrtidae no se conoce bien, pero también parece indicar que la diversidad del grupo en esta área es importante, ya que de 22 individuos recolectados se lograron 17 especies, 6 determinadas, lo que hace de esta región un área prioritaria para la conservación. En 1958, en la cuenca ribereña que recarga el acuífero costero de Oaxaca, había claros menores al 5\% en el bosque tropical seco, donde se sembraban los tradicionales maizales, cultivos de fríjol y árboles frutales. Cuarenta años después la cubierta forestal se redujo en un $50 \%$; únicamente el $20 \%$ se mantiene con la semblanza anterior, mientras que el resto sufrió la extracción parcial de sus recursos madereros (Barkin y Paillés, 2008). Durante los últimos 15 años, el nivel de deforestación ha sido el doble que en los 25 años previos. Parte importante de esta destrucción la aprovecha el sistema de "milpa caminante" (la roza-tumba-quema que se realiza derribando el bosque para cosechar maíz y sus cultivos asociados por unos cuantos años).

A la devastación de los bosques ha seguido la erosión, teniendo como resultado un final crítico: el agua necesaria para proveer el área de desarrollo turístico de Bahías de Huatulco se agotará para el año 2020, a menos que se implante algún programa de regeneración (Barkin y Paillés, 2008). Estos bosques tropicales secos son de los más frágiles del mundo y están desapareciendo rápidamente junto con su inmensa biodiversidad, mucha aún sin ser descubierta. Dada esta perspectiva, los autores están de acuerdo en el desarrollo de programas urgentes para la conservación de áreas protegidas y no protegidas de Huatulco.

Finalmente, se considera la conveniencia de un estudio a largo plazo, usando más métodos de recolección para probar la hipótesis basada en los métodos no paramétricos que indican que de $45 \%$ a $63 \%$ de la fauna de encírtidos no ha sido aún recolectada.

Diversidad de Hymenoptera. González-Hernández (1997) realizó un inventario sobre los himenópteros parasitoides mexicanos, donde se registra la presencia de 11 familias reunidas en 4 superfamilias, tomando los datos de 7 colecciones: Biological Resources Division Center(BRDC), Florida State Collection of Agriculture (FSCA), Texas A\&M University (TAMU), Universidad Autónoma de Nuevo León (UANL), Universidad Autónoma de Tamaulipas (UAT), University of California, Riverside (UCR), United Status Nacional Museum (USNM). Las familias de Apocrita Parasitica recolectadas en este proyecto fueron 25, reunidas en 7 superfamilias; sólo para la localidad de Huatulco, el registro de familias fue más del doble que el contenido en dicho catálogo.

Ruiz Cansino y Coronado Blanco (2005) mencionan el registro de 49 familias de Hymenoptera en la Reserva de la Biosfera El Cielo, Tamaulipas (RBC), es decir el 61\% del total de las familias de Hymenoptera, comparando con Borror et al. (1989); la misma comparación realizada en la selva baja caducifolia de Huatulco, Oaxaca registró un $57 \%$ del total de las familias de Hymenoptera. RodríguezPalafox y Corona (2002) listaron los artrópodos de la región de Chamela, Jalisco, México, una localidad con BTC; la lista incluye los artrópodos que han sido registrados en 96 artículos, publicados entre 1975 y 1999, donde se registraron 10 familias de Hymenoptera, la mayor parte pertenecientes a Apoidea; 6 de estas familias también se registrarón en Huatulco, Oaxaca.

Portuondo Ferrer y Fernández Triana (2004) trabajaron con la diversidad de los himenópteros de los macizos montañosos de Cuba oriental, registrando para ello 44 familias, donde Formicidae, al igual que en nuestro proyecto, fue la familia más abundante. Ichneumonidae, la más rica en especies, pero poco abundante en Cuba, fue de las más abundantes en Huatulco. Se considera que su poca abundancia en Cuba se debió al método de muestreo. En Cuba no se usaron las trampas Malaise, un excelente método de recolección de ichneumónidos. Con el resto de las familias hubo diferencias más significativas, en cuanto a abundancias se refiere, que pudieron darse por diversos factores, desde la diferencia en biomas hasta los métodos de recolección utilizados.

A pesar de que este trabajo ofrece sólo una aproximación preliminar de la extraordinaria diversidad del orden Hymenoptera en los bosques tropicales secos de México, 
puede constituir un punto de partida para la protección de estos insectos en estas áreas naturales.

\section{Agradecimientos}

A Felipe Noguera, Enrique González y Enrique Ramírez, por permitirnos usar el material recolectado durante su proyecto. Al Instituto de Biología de la UNAM y a su directora Dra. Tila Ma. Pérez Ortiz, así como al posgrado en Ciencias Biológicas, UNAM y a CONACYT por todo el apoyo recibido.

\section{Literatura citada}

Barkin, D. y C. Paillés. 2008. Colaboración con la comunidad para el ecoturismo: una estrategia de gestión sustentable de recursos regionales. http://www.uaemex.mx/plin/psus/rev2/ b02.html. Consultada: julio, 2008.

Borror, D. J., C. A. Triplehorn and N. F. Johnson. 1989. An introduction to the study of insects. Saunders College, Philadelphia. $875 \mathrm{p}$.

Ceballos, G. y A. García. 1995. Conserving neotropical biodiversity: the role of dry forests in western Mexico. Conservation Biology 9:1349-1356.

Celso, F. M. 2002. Diversity of the bee fauna of the Brazilian Caatinga. Pollinating bees. The conservation link between Agriculture and Nature - Ministry of Environment, Brasilia. p. 131-134.

Chazdon, R . L., R. K. Colwell, J. S. Denslow y M. R. Guariguata. 1998. Statistical methods for estimating species richness of woody regeneration in primary and secondary rain forest of Northeastern Costa Rica. In Forest biodiversity research, monitoring and modeling. Conceptual background and old world case studies, F. Dallmeier y J. A. Comiskey (eds.). UNESCO and The Parthenon, Paris. p. 285-309.

Colwell, R. K. 2006. EstimateS, Version 8.0: Statistical Estimation of Species Richness and Shared Species from Samples (Software and User's Guide). Freeware for Windows and Mac OS. http://viceroy.eeb.uconn.edu/estimates. Consultada: junio, 2008.

Collwell, R. K.y J. A Coddington. 1994. Estimating terrestrial biodiversity through extrapolation. Philosophical Transactions of the Royal Society (Series B) 345:101-118.

De Santis, L. 1963. Encírtidos de la República Argentina (Hymenoptera: Chalcidoidea). Anales de la Comisión de Investigación Científica, IV. La Plata. Buenos Aires. 398 p.

García, E. 1981. Modificaciones al sistema de clasificación climática de Köppen. Instituto de Geografía, UNAM, México, D.F. 246 p.

González-Hernández, A. 1997. Inventario de Hymenoptera Parasitica en México. Laboratorio de Entomología y Artrópodos,Facultad de Ciencias Biológicas, Universidad Autónoma de Nuevo León, Monterrey. http://www.conabio. gob.mx/institucion/proyectos/resultados/InfP021.pdf.
Consulta: agosto, 2008.

Hammer, Ø; D. A. T. y P. D. Ryan. 2001. PAST: Paleontological statistics software package for education and data analysis. Paleontología Electrónica 4: 9 p. http://palaeo-electronica. org/2001_1/past/issue1_01.htm. Consulta: julio, 2008.

Janzen, D. H. 1988. Tropical dry forest. The most endangered major tropical ecosystem. In Biodiversity, E. O. Wilson (ed.). National Academy, Washington, D. C. p. 130-137.

Kitching, R. L., D. Li y N. E Stork. 2001. Assessing biodiversity 'sampling packages': how similar are arthropod assemblages in different tropical rainforest. Biodiversity and Conservation 10:793-813.

Moreno, C. E. 2001. Métodos para medir la biodiversidad. CYTED, ORCYT/UNESCO y SEA (eds.). Zaragoza. $150 \mathrm{p}$.

Noyes, J. S. 1980. A review of the genera of Neptropical Encyrtidae (Hymenoptera: Chalcidoidea). Bulletin of the British Museum (Natural History) 41: 3.

Noyes, J. S. 1982. Collecting and preserving chalcid wasps (Hymenoptera: Chalcidoidea). Journal of Natural History 16:315-334.

Noyes, J. S. 1989. The diversity of Hymenoptera in the tropics, with special reference to Parasitica in Sulawesi. Ecological Entomology 14:197-207.

Noyes, J. S. 2008. Universal Chalcidoidea Database. The Natural History Museum, London. http://www.nhm.ac.uk/researchcuration/projects/chalcidoids/. Consulta: agosto, 2008.

Noyes, J. S. y M. Hayat. 1994. Oriental Mealy $\underline{b} u g$ Parasitoids of the Anagyrini (Hymenoptera: Encyrtidae). CABI, London. $554 \mathrm{p}$.

Noyes, J. S., J. B. Woolley and G. Zolnerowich. 1997. Family Encyrtidae. Chapter 8. In Annotated keys to the genera of Nearctic Chalcidoidea (Hymenoptera), G. A. P. Gibson, J. T. Huber y J. B. Woolley (eds.). National Research Council Canada, NRC Research Press, Ottawa. p. 170-320.

Portuondo Ferrer, E. y J. L. Fernández Triana. 2004. Biodiversidad del orden Hymenoptera en los macizos montañosos de Cuba oriental. Boletín de la Sociedad Entomólogica Aragonesa 35:121-136. http://dialnet.unirioja.es/servlet/ articulo?codigo=1047880. Consulta: julio, 2008.

Rodríguez-Palafox, A. y Corona A. M. 2002. Lista de artrópodos de la región de Chamela, Jalisco, México. In Historia Natural de Chamela, F. A. Noguera, J. H. Vega Rivera, A. N. García Aldrete y M. Quesada Avendaño (eds.). Instituto de Biología, UNAM, México, D.F. p. 203-232.

Rodríguez-Velez B. y J. B. Woolley, 2005. La fauna de la familia Encyrtidae (Hymenoptera: Chalcidoidea) en el bosque tropical caducifolio de la sierra de Huautla, Morelos, México, D.F. Folia Entomológica Mexicana 44 (Supl.):147-155.

Ruiz C. E. y J. M. Coronado B. 2005. Hymenoptera In Historia Natural de la Reserva de la Biosfera El Cielo, R.G., P.Sánchez, C.Reyes y R. Dirzo, (eds). Universidad Autónoma de Tamaulipas/ Instituto de Ecología, A.C./ Instituto de Ecología, UNAM, México, D.F. p. 445-453.

Rzedowski, J. 1991. Diversidad y orígenes de la flora fanerogámica de México. Acta Botanica Mexicana 14:321.

Steyskal, G. C. 1981. A bibliography of the malaise trap. Proceedings of the Entomological Society of Washington 
$83: 225-229$.

Townes, H. 1972. A light-weight Malaise trap. Entomological News 83:239-247.

Trejo, I. 1998. Distribución y diversidad de selvas bajas de México: relaciones con el clima y el suelo. Tesis doctorado, Facultad de Ciencias, UNAM, México, D. F. 210 p.

Trjapitzin,V. A. y E. Ruiz-Cancino. 1995. Annotated check-list of encyrtids (Hymenoptera: Chalcidoidea: Encyrtidae) of Mexico. Folia Entomológica Mexicana 94:7-32.

Wilson, E. O. 1988. The current state of biological diversity. In Biodiversity, E. O. Wilson (ed.). National Academic, Washington, D.C. p. 3-18.

Wolda, H. 1983. Diversity, diversity indices and tropical cockroaches. Oecologia (Berlin) 58:290-298.

Apéndice 1. Ejemplares de Hymenoptera recolectados en bosque tropical caducifolio, Huatulco, Oaxaca, México.

\begin{tabular}{|c|c|c|c|c|}
\hline Suborden y superfamila & Familia & Núm./(\%) & Mes & $\begin{array}{c}\text { Núm. de } \\
\text { individuos/mes }\end{array}$ \\
\hline SYMPHYTA & & $19(1.13 \%)$ & & \\
\hline \multirow[t]{7}{*}{ Tenthredinoidea } & & $19(1.13 \%)$ & & \\
\hline & Argidae & $2(0.11 \%)$ & febrero & 1 \\
\hline & & & agosto & 1 \\
\hline & Pergidae & $17(1.01 \%)$ & julio & 9 \\
\hline & & & agosto & 6 \\
\hline & & & octubre & 1 \\
\hline & & & noviembre & 1 \\
\hline APOCRITA & & $1443(86 \%)$ & & \\
\hline (ACULEATA) & & $1007(60 \%)$ & & \\
\hline Apoidea & & $44(2.6 \%)$ & & \\
\hline \multirow[t]{13}{*}{ (Apiformes) } & & $37(2.20 \%)$ & & \\
\hline & Anthophoridae & $5(0.3 \%)$ & $\begin{array}{l}\text { enero } \\
\text { octubre }\end{array}$ & $\begin{array}{l}1 \\
4\end{array}$ \\
\hline & Apidae & $24(1.4 \%)$ & febrero & 3 \\
\hline & & & marzo & 2 \\
\hline & & & abril & 13 \\
\hline & & & junio & 1 \\
\hline & & & julio & 2 \\
\hline & & & octubre & 1 \\
\hline & & & noviembre & 2 \\
\hline & Halictidae & $8(0.47 \%)$ & febrero & 3 \\
\hline & & & marzo & 2 \\
\hline & & & $\begin{array}{l}\text { jul10 } \\
\text { octubre }\end{array}$ & 1 \\
\hline & & & diciembre & 1 \\
\hline \multirow[t]{5}{*}{ (Spheciformes) } & & $7(0.41 \%)$ & & \\
\hline & Ampulicidae & $4(0.23 \%)$ & octubre & 4 \\
\hline & Crabronidae & $2(0.11 \%)$ & febrero & 1 \\
\hline & & & marzo & 1 \\
\hline & Pemphredonidae & $1(0.06 \%)$ & julio & 1 \\
\hline \multirow[t]{22}{*}{ Chrysidoidea } & & $158(9.4 \%)$ & & \\
\hline & Bethylidae & $87(5.17 \%)$ & febrero & 16 \\
\hline & & & marzo & 7 \\
\hline & & & abril & 13 \\
\hline & & & junio & 5 \\
\hline & & & julio & 18 \\
\hline & & & agosto & 9 \\
\hline & & & $\begin{array}{l}\text { septiembre } \\
\text { octubre }\end{array}$ & $\begin{array}{c}3 \\
10\end{array}$ \\
\hline & & & noviembre & 6 \\
\hline & Chrysididae & $2(0.11 \%)$ & marzo & 1 \\
\hline & & & octubre & 1 \\
\hline & Dryinidae & $66(3.9 \%)$ & febrero & 5 \\
\hline & & & marzo & 3 \\
\hline & & & abril & 1 \\
\hline & & & junio & 12 \\
\hline & & & julio & 11 \\
\hline & & & agosto & 11 \\
\hline & & & septiembre & 1 \\
\hline & & & $\begin{array}{c}\text { octubre } \\
\text { diciembre }\end{array}$ & $\begin{array}{c}19 \\
3\end{array}$ \\
\hline & Sclerogibbidae & $3(0.17 \%)$ & febrero & 1 \\
\hline & & & abril & 1 \\
\hline & & & noviembre & 1 \\
\hline
\end{tabular}


Apéndice 1. Continúa.

Suborden y superfamila

Vespoidea

(PARASITICA)

Ceraphronoidea

Chalcidoidea

\section{Familia}

Formicidae

Mutillidae

Pompilidae

Rhopalosomatidae

Vespidae

Sapygidae

Scoliidae

Sierolomorphidae

Tiphiidae

Ceraphronidae

Megaspilidae

Aphelinidae

Chalcididae

Elasmidae
Núm./(\%)

$1024(61 \%)$

$653(39 \%)$

$62(3.7 \%)$

$235(13.4 \%)$

$17(1.01 \%)$

$1(0.06 \%)$

$2(0.11 \%)$

$1(0.06 \%)$

$14(0.83 \%)$

$39(2.32 \%)$

$436(26 \%)$

$7(0.41 \%)$

$6(0.35 \%)$

$1(0.06 \%)$

$120(7.13 \%)$

$1(0.06 \%)$

$10(0.6 \%)$

$1(0.06 \%)$
Mes

Núm de individuos/mes

$\begin{array}{cc}\text { enero } & 49 \\ \text { febrero } & 71 \\ \text { marzo } & 87 \\ \text { abril } & 15 \\ \text { junio } & 41 \\ \text { julio } & 40 \\ \text { agosto } & 31 \\ \text { septiembre } & 30 \\ \text { octubre } & 28 \\ \text { noviembre } & 9 \\ \text { diciembre } & 11 \\ \text { enero } & 1 \\ \text { febrero } & 4 \\ \text { marzo) } & 4 \\ \text { abril } & 4 \\ \text { junio } & 8 \\ \text { julio } & 35 \\ \text { agosto } & 2 \\ \text { octubre } & 3 \\ \text { diciembre } & 1\end{array}$

diciembre

febrero

marzo

abril

junio

julio

agosto

septiembre

octubre

noviembre
diciembre

junio

agosto

octubre

noviembre

diciembre

enero

septiembre

octubre

enero

febrero

marzo

abril

julio

octubre

enero

febrero

marzo

abril)

junio

octubre

diciembre

49

71

87

41

31

28

9

1

4

35

3

12

24

10

29

11

44

32

6

2

1

4

1

1

1

1

2

3

5
3

12

2

3

8

2
10

$\begin{array}{cc}\text { junio } & 1 \\ \text { julio } & 4 \\ \text { agosto } & 1 \\ \text { agosto) } & 1 \\ & \\ \text { febrero } & 1 \\ \text { febrero } & 1 \\ \text { agosto } & 3 \\ \text { octubre } & 3 \\ \text { diciembre } & 3 \\ \text { diciembre } & 1\end{array}$


Apéndice 1. Continúa.

Suborden y superfamila

\section{Cynipoidea}

Evanioidea

Ichneumonoidea

Familia
Encyrtidae
Eucharitidae
Eulophidae

Eulophidae

Eupelmidae

Eurytomidae

Mymaridae

Perilampidae

Pteromalidae

Signiphoridae

Tetracampidae

Torymidae

Trichogrammatidae

$3(0.17 \%)$

$1(0.06 \%)$

$3(0.17 \%)$

$6(0.35 \%)$

$6(0.35 \%)$

Eucoilidae

$6(0.35 \%)$

$16(0.95 \%)$

$2(0.11 \%)$

$14(0.83 \%)$

Evaniidae

Aulacidae

$201(11.95 \%)$

\begin{tabular}{|c|c|}
\hline Mes & $\begin{array}{l}\text { Núm. de } \\
\text { individuos/mes }\end{array}$ \\
\hline febrero & 5 \\
\hline marzo & 3 \\
\hline abril & 1 \\
\hline junio & 4 \\
\hline julio & 3 \\
\hline agosto & 1 \\
\hline septiembre & 1 \\
\hline $\begin{array}{l}\text { octubre } \\
\text { diciembre }\end{array}$ & 2 \\
\hline diciembre & 2 \\
\hline diciembre & 1 \\
\hline marzo & 1 \\
\hline junio & 1 \\
\hline julio & 4 \\
\hline agosto & 3 \\
\hline septiembre & 2 \\
\hline octubre & 1 \\
\hline febrero & 5 \\
\hline marzo & 1 \\
\hline abril & 1 \\
\hline septiembre & 1 \\
\hline octubre & 3 \\
\hline noviembre & 1 \\
\hline febrero & 2 \\
\hline junio & 1 \\
\hline septiembre & 4 \\
\hline diciembre & 1 \\
\hline febrero & 2 \\
\hline marzo & 1 \\
\hline junio & 1 \\
\hline octubre & 1 \\
\hline enero & 1 \\
\hline febrero & 6 \\
\hline marzo & 1 \\
\hline abril & 2 \\
\hline junio & 13 \\
\hline julio & 6 \\
\hline agosto & 4 \\
\hline octubre & 1 \\
\hline $\begin{array}{l}\text { septiembre } \\
\text { octubre }\end{array}$ & $\begin{array}{l}1 \\
2\end{array}$ \\
\hline agosto & 1 \\
\hline junio & 1 \\
\hline julio & 1 \\
\hline septiembre & 1 \\
\hline febrero & 1 \\
\hline abril & 1 \\
\hline agosto & 3 \\
\hline octubre & 1 \\
\hline
\end{tabular}

julio

noviembre

marzo

junio

octubre 
Apéndice 1. Continúa.

Suborden y superfamila

Platygastroidea

Proctotrupoidea

\section{Familia \\ Braconidae}

Ichneumonidae

Scelionidae

Platygastridae
Núm./(\%)

$92(5.47 \%)$

$74(4.4 \%)$

$25(1.48 \%)$

$49(2.91 \%)$

$12(0.71 \%)$

$12(0.71 \%)$

$\begin{array}{cc}\text { Mes } & \begin{array}{c}\text { Núm. de } \\ \text { individuos/mes }\end{array} \\ \text { enero } & 7 \\ \text { febrero } & 12 \\ \text { marzo } & 4 \\ \text { abril } & 4 \\ \text { junio } & 3 \\ \text { julio } & 32 \\ \text { agosto } & 18 \\ \text { septiembre } & 1 \\ \text { octubre } & 7 \\ \text { noviembre } & 2 \\ \text { diciembre } & 2 \\ \text { enero } & 9 \\ \text { febrero } & 3 \\ \text { julio } & 28 \\ \text { agosto } & 14 \\ \text { septiembre } & 8 \\ \text { octubre } & 29 \\ \text { noviembre } & 2 \\ \text { diciembre } & 16 \\ & \\ \text { febrero } & 1 \\ \text { junio } & 6 \\ \text { julio } & 14 \\ \text { agosto } & 3 \\ \text { octubre } & 1 \\ \text { febrero } & 5 \\ \text { marzo } & 1 \\ \text { abril } & 6 \\ \text { junio } & 7 \\ \text { julio } & 15 \\ \text { agosto } & 8 \\ \text { septiembre } & 2 \\ \text { octubre } & 3 \\ \text { noviembre } & 1 \\ \text { diciembre } & 1 \\ & \\ \text { julio } & 7 \\ \text { agosto } & 2 \\ \text { octubre } & 3 \\ & \end{array}$

